\title{
Variations
}

Variations

Revue internationale de théorie critique

\section{Numérique et libération de la production}

\section{scientifique}

Critique de l'économie politique de l'Open Access

Christophe Magis et Fabien Granjon

\section{(2) OpenEdition}

\section{Journals}

Édition électronique

URL : http://journals.openedition.org/variations/733

DOI : 10.4000/variations.733

ISSN : 1968-3960

Éditeur

Les amis de Variations

Référence électronique

Christophe Magis et Fabien Granjon, « Numérique et libération de la production scientifique »,

Variations [En ligne], 19 | 2016, mis en ligne le 06 avril 2016, consulté le 30 avril 2019. URL : http://

journals.openedition.org/variations/733; DOI : 10.4000/variations.733

Ce document a été généré automatiquement le 30 avril 2019.

Les ami•e•s de Variations 


\title{
Numérique et libération de la production scientifique
}

\author{
Critique de l'économie politique de l'Open Access
}

Christophe Magis et Fabien Granjon

\begin{abstract}
«Travailler sur des projets liés au libre accès, s'est se retrouver accusé, un jour d'être un vendu aux principes du néolibéralisme et, le lendemain, d'être un marxiste anti-corporatiste.» Cameron Neylon, cité par Martin Eve, Open Access and the Humanities. «Il arrive [...] que la machine, ce plus puissant moyen d'abréger le travail, devient le plus sûr moyen de transformer la vie entière du travailleur et de sa famille en temps de travail disponible pour la mise en valeur du capital [...]. » Friedrich Engels, Socialisme utopique et socialisme scientifique.
\end{abstract}

1 À mesure que les technologies numériques de l'information et de la communication (TNIC) se déploient dans le champ scientifique (recherche et enseignement supérieur), impulsant, notamment au sein des humanités, de «nouveaux modes de travail», de «nouveaux lieux de production » et de « nouveaux publics» (Citton, $2015: 172)$, plusieurs débats émergent quant au sens d'ensemble et à la direction à donner à ces mutations : « À l'heure du cloud, du software as a service (SAS), du big data et des géants mondiaux du numérique, il semble impossible d'éviter le débat sur les initiatives [...] en matière d'infrastructures numériques de recherche » (Dacos, 2014). C'est le cas notamment en ce qui concerne la problématique de l'open Access, enjeu majeur des mutations numériques de l'université, actuellement très présente dans les discussions institutionnelles, et tout particulièrement en France. Elle y joue, en effet, un rôle central dans la mesure où elle se trouve au cœur de la loi " pour une République numérique », laquelle a été adoptée par 
l'Assemblée nationale en première lecture en janvier 2016, et divise actuellement la communauté universitaire, entre des réactions particulièrement enthousiastes ou au contraire très sceptiques ${ }^{1}$.

Comme pour un ensemble de catégories récentes liées aux enjeux ouverts par le digital turn, les réalités recouvertes ou à faire recouvrir par la notion d'Open Access ne sont pas tout à fait stabilisées (Suber, 2012), d'autant que sa traduction, en français notamment, fait encore largement débat. "Accès ouvert ", « libre accès ", etc., certaines propositions vont ainsi jusqu'à proposer le terme de « science ouverte " (Farchy et al., 2010 : 10 ; Aigrin, 2011)2. De manière succincte, "le terme "Open Access" fait référence au retrait des barrières d'accès à la recherche scientifique que représentent le prix et les permissions. L' Open Access concerne les travaux de la recherche scientifique évalués par les pairs que l'on peut lire gratuitement en ligne et que quiconque peut redistribuer et réutiliser moyennant le respect de quelques restrictions » (Eve, 2014:1). Facilitée par le processus de numérisation du signe, la circulation de l'information scientifique semble s'affranchir d'un certain nombre de contraintes que faisaient peser sur elle la matérialité particulière des revues papier - autour de laquelle s'étaient ancrés des fonctionnements économiques particuliers et des usages spécifiques. Sous ces nouvelles conditions, se trouvent alors réinterrogés les modes de fonctionnement de la recherche scientifique qui semblent promettre de nouveaux usages sociaux de la science (Vecam, 2005 ; 2011). Ceuxci sont pensés comme pouvant compléter le mouvement de démocratisation de l'enseignement supérieur du dernier tiers du vingtième siècle, lequel était néanmoins contredit par la maigre diffusion de la littérature scientifique et son renforcement par la " crise des périodiques scientifiques ${ }^{3}$ ». Par ailleurs, ici comme dans d'autres secteurs de la culture et des médias, cette période de "crise ", qui résonne de manière cinglante en parallèle des tremblements majeurs de l'économie mondialisée, depuis le début des années 2000 (éclatement de la «bulle internet», «junk bonds», crise économique généralisée), est également perçue, par d'aucuns, comme un porteuse d'opportunités ouvrant la voie à une réflexion d'ensemble pour un partage plus égalitaire des ressources, notamment de nature informationnelle et culturelle.

Outre la question de la nécessité d'un usage généralisé de l'open Access au sein de l'université et des organismes de recherche, les discussions portent également sur les modalités pratiques de sa mise en place. C'est d'ailleurs probablement sur ce point que les tensions sont les plus fortes (Chartron, 2016). Ainsi, le débat actuel voit, y compris dans les rangs des défenseurs de l'open Access, des discours très contradictoires sur la philosophie qui devrait animer sa mise en place et ses formes de financement. Il n'est d'ailleurs pas rare que les participants à ces discussions, d'un bord ou d'un autre, parlent d'«idéologie» (Wolton, 2010; Harnad, 2015; Minard, 2015; Minon et al., 2015) pour désigner les positions qui leur sont opposées, sans qu'on sache véritablement toujours quelles réalités inclure derrière ces formes de déqualification. Dans ce débat qui peut parfois paraître très "pratico-technique ", il faut évidemment ne pas perdre de vue que ce sont des visions différentes de la science et de la société qui s'affrontent. Cet article vise ainsi à interroger les différentes propositions concernant les modalités actuelles de mise en place du libre accès, en analysant à quelles visions ou "philosophies » elles répondent. Nous allons notamment considérer la manière dont le débat sur l'Open Access pourrait être institué en un enjeu politique à même de viser une libération du travail en général, à partir d'une libération du travail scientifique. Pour ce faire, il convient alors de 
réordonner la hiérarchie des principes qui sont à l'origine des réflexions sur l'accès libre, et ce, depuis une critique matérialiste de l'économie politique.

\section{Les trois impulsions majeures en direction de l'Open Access}

4 Nous voudrions, dans cette première partie, analyser les bassins scientifiques et épistémologiques d'émergence des problématiques liées au libre accès. Si ce dernier semble aujourd'hui relativement confus, voire contradictoire, quant à ses buts, ses marges et ses domaines d'application, c'est aussi parce qu'il a vu son apparition dépendre de philosophies différentes, articulées à des revendications variées quant au travail propre à la recherche scientifique. Nous verrons notamment trois impulsions en direction du libre accès, s'articulant à des visées différentes : d'abord une visée scientifique, ensuite une visée d'ordre éthico-politique et une visée plus critique, articulée à une critique de l'économie politique à l'ère numérique.

5 Ce n'est probablement pas à propos des sciences humaines et sociales (SHS) que la nécessité d'une ouverture de l'accès aux principaux outputs de la production scientifique s'est premièrement faite sentir. S'articulant davantage autour d'une culture de la monographie, ou de l'ouvrage scientifique, les SHS ne se sont intéressées que plus tardivement au libre accès (Eve, 2014). Ce n'est pas non plus autour d'une tentative de réponse à la crise des périodiques scientifiques ou d'une critique des modèles économiques de ces derniers que les premières initiatives en direction de l'archive ouverte, puis du libre accès ont été impulsées. Cette critique émerge plus tard dans le débat, notamment impulsée par l'entrée des SHS dans les problématiques liées à l'accès ouvert, en s'articulant à d'autres nécessités, notamment de dissémination scientifique. On considère ainsi que la première archive pleinement ouverte et accessible en ligne a été le projet arXivi ${ }^{4}$ d'après la création en 1991 d'une base de données en ligne archivant et recensant un ensemble de prépublications (preprints), mises à disposition par plusieurs spécialistes d'un sous-champ de la physique afin d'assurer une circulation plus rapide de leurs travaux, dans un secteur scientifique caractérisé par une tradition d'échange régulier des prépublications entre chercheurs (Ginsparg, 1994; Mele, 2010). La base de données a été ensuite ouverte à d'autres domaines de la physique ainsi qu'aux mathématiques, à l'informatique, la biologie et la finance "quantitative", dans une tentative de poursuivre cette accélération de la diffusion de l'information scientifique entre chercheurs, plutôt que de produire une alternative à l'économie des publications scientifiques. D'autres propositions se feront en suivant la même logique, comme par exemple le projet NetEc en économie (1993), devenu RePEc en 1997 (Krichel, 2000).

S'il est, dans ces projets une critique du système des revues scientifiques, elle n'est pas directement orientée vers leur économie, mais vers leur capacité à remplir leur fonction de médiation au sein du monde scientifique :

«Pour les économistes comme pour les physiciens, les revues ne jouent plus depuis longtemps le rôle de vecteur de l'information scientifique qu'elles ont pu remplir dans le passé. Des réseaux d'échanges informels et décentralisés entre chercheurs au sein de communautés spécialisées ont profité de la démocratisation des moyens de communication pour se développer et remplir à leur tour ces fonctions beaucoup plus efficacement que le système traditionnel de publication en revues » (Mounier, 2010a : 26). 
7 Telle décentralisation de la médiation de l'information scientifique permet par ailleurs de déjouer les travers des temps de publication des revues dans des domaines où les découvertes sont rapides: le dépôt dans ces bases de données spécialisées permet aux articles de commencer à être considérés et cités quelquefois plusieurs années avant leur publication effective (Gentil-Beccot et al., 2010). Ces « réseaux d'échange » ont quelquefois donné lieu à des revues en accès ouvert permettant à la fois la publication des articles et la conservation des commentaires des pairs (peer commentary). C'est le cas par exemple de la revue canadienne Surface, dirigée par Jean-Claude Guédon, ou de la revue Psyquoloquy, soutenue par l'American Psychological Association, très pro-active dans le domaine de la publication en ligne, mettant en avant les atouts de cette dernière quant à la rapidité de diffusion des connaissances et la génération de la discussion scientifique ${ }^{5}$ (Harnad, 1991). Ici encore, si le mouvement est largement impulsé par les manières de faire des sciences dures, des techniques et de la médecine (STM), il est tout de même largement suivi par des revues de SHS, notamment à partir du début des années 2000.

Une tout autre philosophie innerve un ensemble d'initiatives qui fleurissent au long des années 1990. Ces initiatives expriment à la fois des considérations scientifiques, mais aussi et surtout politiques et éthiques. Elles sont également, souvent, le fait de domaines des sciences de la nature, notamment des sciences du vivant et de la médecine, "en raison en particulier des problématiques éthiques qui imprègnent fortement les activités liées à la santé » (Mounier, 2010a : 24). Elles font ainsi intervenir à la fois des acteurs du monde scientifique, mais aussi des militants sur des sujets que les institutions scientifiques et politiques reprennent, comme par exemple dans le cas de l'appel du comité américain de la recherche sur l'environnement et les ressources naturelles du conseil national de la science et de la technologie qui pose, dès 1992, la nécessité "d'établir un système d'information des données sur le changement global (GCDIS) qui rassemblerait toutes les données concernant le changement global dans un seul système " (US Congress-OTA, $1994: 21$ ). La question de l'accès facilité à ces données devient d'autant plus centrale, dès lors que, en 1995, le comité sur les données géophysiques et environnementales du même conseil appelle à un échange « libre et sans restriction » des informations concernant le climat, les données météorologiques et atmosphériques, réclamant que «les États-Unis prennent les dispositions nécessaires pour encourager le principe d'un accès libre et ouvert $»^{6}$. En 1996, l'accord des Bermudes (Bermuda Principles) présente un consensus de la communauté scientifique sur le principe d'un accès ouvert aux informations produites suite aux expériences sur le génome humain dans des bases de données publiques. Deux ans plus tard, la « Déclaration de San Jose sur la bibliothèque virtuelle de la santé », qui fait suite à une conférence au Costa Rica du congrès panaméricain de l'information sur les sciences médicales, appelle à un accès libre aux connaissances médicales. Elle est suivie en 2001 d'une seconde déclaration allant dans le même sens. Ces appels s'inscrivent en réalité dans une longue tradition qui oscille entre la peur d'une divulgation trop facile de données scientifiques présentant un intérêt économique, sanitaire ou militaire/stratégique et la certitude de la nécessité de la circulation des résultats scientifiques, notamment aux États-Unis. Charles Vest (2006) rappelle, à ce propos, que même en pleine période de guerre froide, les politiques scientifiques américaines ont maintenu l'idée que pour être bénéfiques « les découvertes fondamentales de la recherche devaient pouvoir circuler librement " (Scientific Research Board, 1947, cité in Vest, 2006). L'administration Reagan rappelle par exemple que « dans la plus grande mesure possible, les produits de la recherche fondamentale doivent voir 
leur accès rester libre » (National Security Decision Directive 189, 1985, cité in Vest, 2006), et que leur éventuelle restriction pour motif d'ordre sécuritaire doit faire l'objet d'une classification.

Pour bon nombre de politiques scientifiques, un accès libre garanti aux découvertes est aussi le meilleur moyen d'éviter les travers de l'espionnage industriel ou militaire qui pourrait déposséder les chercheurs d'un état des recherches que ce même état finance. Ce type de raisonnement anime les initiatives qui émaillent les années 1990 et prévaut lorsque, "[p]eu à peu, les instances nationales et internationales se saisissent du problème et prennent position : l'Unesco en 1999, les Nations Unies en 2000, l'OCDE en 2004 ; voire l'Académie française des sciences en 2001 » (Farchy, Froissart, $2010: 141$ ). Relayées par des mouvements associatifs ou militants, les déclarations qui suivront, dans les années 2000, comme la Budapest Open Access Initiative (2002), le Bethesda Statement on Open Access Publishing (2003) ou la Déclaration de Berlin sur le libre accès à la connaissance (2003) consacrent l'idée que « la recherche sur fonds publics doit revenir au public, à la fois pour des raisons éthiques, sociétales et scientifiques » (Dacos, 2012a : 218), selon une définition du libre accès parfois rassemblée collectivement sous le terme « BBB » (Budapest-Bethesda-Berlin - Suber, 2012; Eve, 2014). Et si, là encore, c'est dans le domaine des sciences de la santé, de la terre et de la matière que la nécessité de l'accès ouvert se fait le plus urgent ${ }^{7}$, les SHS finissent par embrasser le mouvement, notamment avec les réflexions sur les humanités numériques (Dacos, 2014 ; Citton, 2015), même si nous verrons, infra, que la reprise du contrôle sur le débat par les institutions gouvernementales n'est pas sans poser un certain nombre de problèmes.

Il est, en plus de ces deux mouvements, qui mettent en avant des considérations scientifiques, puis éthico-politiques, une troisième impulsion qui tend à faire du libre accès également un outil de critique économique à l'ère du numérique. En suivant Martin Eve (2014), on peut considérer que cette impulsion apparait lorsqu'une critique de la " crise des périodiques scientifiques » rencontre la culture libriste, notamment au sein des SHS. Il faut ainsi, dans un premier temps, reconnaître que cette crise des périodiques, qui a vu, aux États-Unis, les dépenses des bibliothèques pour l'abonnement aux périodiques scientifiques augmenter de près de $400 \%$ entre 1986 et $2011^{8}$, a principalement touché le secteur des STM. Toutefois, outre que certaines disciplines comme l'économie ou la psychologie ont également vu les prix des abonnements à leurs revues les plus importantes augmenter, c'est aussi, selon le principe de vente par «bouquets » mis en place par les principaux éditeurs, le reste des SHS qui a eu à souffrir de cette augmentation, laquelle a même conduit certaines institutions d'enseignement et de recherche parmi les plus fortunées, à l'instar de l'Université de Harvard, à dénoncer une «situation intenable» (Sample, 2012), ne pouvant plus assumer financièrement l'accès à toutes les ressources qui lui étaient nécessaires (Eve, 2014). C'est alors un large mouvement de contestation qui émerge depuis le monde académique américain, à partir notamment des bibliothèques universitaires dans un premier temps, pour s'opposer purement et simplement à ce qu'elles considèrent comme un racket. Puis rapidement, au tournant des années 2000 , des chercheurs viennent prendre part à ce débat, montrant ainsi, par exemple, que la hausse des prix des abonnements aux revues payantes (Toll Access) ne se justifie ni quant à la variation de qualité des papiers dans le temps (Kirillidou, 1999), ni quant à une différence de qualité entre les revues payantes et les revue libres (Bergstrom, 2001). Ce mouvement, rendant de plus en plus visible, au cours de la décennie 2000, le contrôle absolu sur la publication académique et les profits très 
importants des grandes majors internationales dominant un marché très concentré, (notamment RELX', Springer, Wiley-Blackwell et Taylor \& Francis), a engendré un intérêt de plus en plus fort pour l'économie politique de la publication scientifique.

11 Ce mouvement prend place dans un tournant général de l'économie des biens culturels et communicationnels, s'appuyant sur les différents apports de la numérisation et de l'informatisation des sociétés. En effet, le tournant des années 2000 est aussi celui de la crise structurelle qui accompagne les mutations numériques de l'ensemble des industries culturelles (Chantepie, Le Diberder, 2010). Notamment, la numérisation des contenus, combinée à la démocratisation des terminaux informatiques et des offres personnelles d'accès à l'Internet haut-débit, a permis de nouveaux usages et de nouveaux modes de partage de la culture à même de contredire les formes traditionnelles d'appropriation des biens culturels, basées sur un contrôle des droits de copie et de diffusion. Prenant place dans cette période de mutations, la critique scientifique rencontre le mouvement libriste et son ethos centré, comme le rappelle Sébastien Broca (2013), sur la création autonome et libre à base de bidouillage technologique et de philosophie Do It Yourself, ainsi que sur la nécessaire libre circulation de l'information. Cette rencontre autour du libre accès à la culture scientifique a encouragé une réflexion critique sur la recherche de nouveaux modèles sociaux, à même de partir des principes des pratiques universitaires (dont certaines se fondent facilement dans l'ethos libriste), pour aller vers son extension au reste de l'économie. C'est, par exemple, un argument central pour un militant du libre accès comme Peter Suber (2012) : la liberté de la recherche académique vient justement de ce qu'elle ne repose pas sur l'éventuel retour de gains en droit d'auteur pour exister. Selon Eve, cet argument coïncide avec les critiques du dispositif du copyright posées par les défenseurs du logiciel libre qui se traduisent dans une question telle que : " pourquoi les chercheurs devraient-ils conserver la protection économique du copyright s'ils ne dépendent pas du système de rémunération que celui-ci est censé soutenir ?» (2014: 18). Ainsi, constatant que les très gros profits des éditeurs scientifiques sont produits «en parasite », en s'appuyant dans une large mesure sur leur travail, de rédaction mais surtout de révision pour lequel ils ne sont que très rarement rémunérés (Gusterson, 2012), certains chercheurs vont jusqu'à proposer un dépassement de la publication scientifique à même de contredire le seul pouvoir de labellisation qu'apporte l'éditeur scientifique (Apt, 2001).

12 Nous voyons donc bien qu'il y a, au sein du mouvement en faveur de l'Open Access, une dimension intéressante de critique plus large de l'économie politique, à partir d'une critique de l'économie de la publication scientifique. Toutefois, s'articulant autour de trois impulsions, entre lesquelles cette dimension ne semble pas la plus urgente, et peutêtre aussi par son lien même avec la culture libriste, qui n'est pas toujours pensée dans le cadre d'une critique de l'économie politique, le mouvement a du mal à se concentrer sur cet aspect de la critique économique. Ce dernier, se mêlant fréquemment aux autres impulsions, tend alors à être relégué à portion congrue, comme un simple argument servant à encourager les actions proposées par les autres impératifs. C'est notamment le cas en Europe, et particulièrement en France où, il faut bien le reconnaître, les tarifs pratiqués par les éditeurs en SHS autre qu'anglophones, n'ont rien à voir avec ceux des majors de l'édition scientifique dans les pays anglo-saxons (cf. Minard, 2015). Alors, outre qu'ils y rencontrent un grand nombre de détracteurs, les projets de mise en place du libre accès tendent souvent à évacuer complètement cette dimension. 


\section{... Et leur articulation aux impulsions politiques}

13 En effet, pris entre ces différentes impulsions, le libre accès peine à trouver des modalités de mise en place à même de toutes les satisfaire et notamment de porter sérieusement les visées critiques de la troisième. Surtout que ces modalités s'articulent aussi à des impératifs institutionnels, lors de la récupération de la question par les États ou d'autres institutions supranationales, dans un contexte de libéralisation de l'enseignement supérieur et de soumission de la recherche aux nécessités de la croissance économique. Il est alors intéressant d'analyser les visions du libre accès que portent les différents modèles de mise en place actuellement proposés car, majoritairement dominés par ces impératifs institutionnels, les débats autour de l'open Access risquent de perdre leur capacité à laisser entrevoir la possibilité d'une économie réellement alternative.

\section{« Gold Road » et « Green Road »}

Dans les recommandations des déclarations internationales concernant le libre accès, il est généralement exposé deux modèles quant à sa possibilité de mise en place pratique. Le premier et le principal modèle est la "voie dorée " (Gold road) qui consiste à faire supporter les coûts de publication non par les lecteurs (via des systèmes traditionnels d'abonnement), mais par les auteurs eux-mêmes, selon un principe appelé «auteurpayeur $\aleph^{10}$. Il s'agit, pour les éditeurs de simplement renverser la chaîne du financement : ne vendant plus l'accès aux articles, "ils doivent trouver leur rémunération ailleurs " (Eve, 2014 : 9). Bien que largement encouragé par la récente conférence de Berlin sur le libre accès de décembre 2015 (Chartron, 2016), ce modèle est fréquemment critiqué au sein même des partisans du libre accès (Harnad, 2001), notamment en ce qu'il donne la part belle aux chercheurs issus des laboratoires les mieux dotés, et notamment les laboratoires de sciences de la nature (Dacos, 2012a/b ; Mounier, 2010b; Lemercier, 2015). Il n'est donc pas véritablement en mesure de satisfaire les impératifs scientifiques, et encore moins éthico-politiques du libre accès.

Le second modèle, appelé «voie verte " (Green Road) consiste en l'encouragement de l'auto-archivage, par les chercheurs eux-mêmes, de leurs articles dans des bases de données institutionnelles ${ }^{11}$, thématisées ou non. Cette « voie » se situe ainsi directement dans la continuité des projets comme le projet arXiv que nous évoquions supra. Elle pourrait représenter une «transition avantageuse » vers le libre accès (Harnad, 2007) et cohabiter avec l'édition traditionnelle, d'autant qu'«en l'absence de politique institutionnelle encourageant l'auto-archivage, le taux de dépôt spontané est [déjà] de l'ordre de $15 \%$ » (Suber, 2012: 57). Par ailleurs, la plupart des études sur la question concluent généralement que le Green $O A$ n'entraînerait pas pour autant une dislocation de l'économie de la publication (Harnad, 2007 ; Eve, 2014). Cette voie paraît donc convenir aux impératifs scientifiques et éthico-politiques du libre accès, et c'est en s'appuyant sur ces arguments, défendus par bon nombre de militants du libre accès, que les États et institutions publiques ont encouragé sa mise en place. C'est le cas notamment pour la France où, par exemple, l'Agence Nationale de la Recherche (ANR), principale agence publique de financement de la recherche demande, depuis 2007, que "toutes les publications consécutives aux projets financés par elle soient d'ores et déjà intégrées par les chercheurs au système d'archives ouvertes HAL $»^{12}$. Depuis 2010, les universités 
publiques ainsi que les grands établissements de financement de la recherche ont également été incités à être "proactifs » quant au dépôt des productions scientifiques archives ouvertes, en développant leurs propres portails d'accès et d'extraction de HAL; l'archive ouverte française a également développé un service permettant de connaître les politiques des éditeurs concernant l'auto-archivage, afin d'encourager davantage les chercheurs à embrasser cette pratique (Héloise). En juillet 2015, l'organisation européenne Science Europe (regroupant les principales institutions de recherche et les principales agences de financement de la recherche européennes) "se déclare en faveur d'un engagement "diamant" pour l'accès ouvert» (Dacos, 2015), et demande aux éditeurs scientifiques d'autoriser les auteurs à déposer leurs articles dans les bases de données d'accès ouvert.

Toutefois, outre l'intérêt important d'ordre archivistique et la facilité rendue à la consultation des travaux de recherche, à même de servir les impératifs scientifiques et éthico-politique du libre accès, la « voie verte » révèle néanmoins un point de tension qui met au jour la faiblesse, dans les débats, des discours critiques quant à l'impératif économique. Car d'un côté, si les États et les institutions de financement de la recherche poussent pour la mise en place de l'Open Access, c'est bien évidemment aussi pour "permettre à l'industrie de s'approprier les fruits de la recherche scientifique (souvent publique) et de se l'approprier dans une perspective commerciale » (Eve, $2014: 23)$. C'est ainsi un encouragement économique indirect du secteur industriel qui, s'il concerne surtout les STM, ne va pas sans s'accompagner d'un renforcement du contrôle de toute la recherche scientifique. La connaissance et le savoir devenant, au fil du début du XXI siècle, des ressources de plus en plus stratégiques dans une économie s'articulant autour de l'innovation, le libre accès se voit ainsi, dans son rapport aux institutions, inscrire sa « voie verte » dans des perspectives portées par une vision particulièrement libérale.

Par ailleurs, soumise ainsi à la subvention publique, cette vision du libre accès est aussi sujette aux aléas politiques et au désengagement progressif (Dacos, 2012b). Et dans ce cas, il ne faut pas oublier que de nouveaux acteurs du web rivalisent déjà avec les initiatives étatiques ou institutionnelles: des plates-formes privées de réseau social, à destination des universitaires ou des jeunes chercheurs comme Academia, ResearchGate ou Mendeley. Fonctionnant comme agents d'une « classe vectorialiste » (Wark, 2006), « qui profitent de la nécessaire vectorialisation matérielle de l'information » (Citton, 2013 : 168), ces acteurs attirent par un accès gratuit aux ressources dissimulant le fond véritable de l'exploitation, selon un principe analogue aux user generated contents de l'économie du "web collaboratif» (Bouquillion, Matthews, 2010). Aussi, serait-il intéressant de comprendre ce que de tels modèles de la recherche véhiculent comme représentations du travail scientifique. Derrière un « unexpected reader ${ }^{13}$ " (Smith, 2011, cf. Granjon, Magis dans ce numéro) devenant la figure idéal-typique de l'usager de la plate-forme scientifique, émerge une figure assez "expected»: le précaire scientifique dont les activités relèvent assez majoritairement de formes plus ou moins complexes de travail temporaire généralement mal rémunéré, voire gratuit (Fuchs, 2014a; Hesmondhalgh, 2010 ; Terranova, 2004). Un tel modèle socio-économique postule ainsi un usager dont il est attendu qu'il soit en capacité de s'informer, faire le tri dans l'abondance des données qui lui sont proposées « gratuitement » (selon le principe du libre accès) et de se montrer capable de produire de l'information appropriable par des plates-formes spécialisées qui en tireraient bénéfices pour entretenir le libre accès lui-même. C'est ici que la figure du lecteur rejoint celle du digital labourer. Car si l'on en reste à la figure mythique originale 
de l'unexpected reader, il s'agit bien de l'exploitation par une revue d'un travail réalisé par une personne hors de toute rémunération (hormis une rétribution symbolique selon un principe qui n'est pas sans rappeler certaines formes de travail culturel - Hesmondhalgh, 2010). Il s'agit là d'une situation assez proche de ce qu'analysent certains spécialistes du digital labour, laquelle induit des formes d'exploitation de travail gratuit, quand bien même celui-ci n'est pas toujours considéré comme tel, les formes idéologiques du numérique cachant par exemple « l'exploitation sous l'apparence du jeu » (Fuchs, 2014b ; Scholz, 2013).

Face à ces travers, qui touchent à la fois la "voie verte" et la "voie dorée », les détracteurs du libre accès, notamment chez les directeurs de certaines revues veillent et militent pour un maintien du statu quo, et notamment que les délais d'embargo (i.e. l'intervalle de temps à considérer avant d'accorder l'autorisation de dépôt dans les bases de données) soient assez longs. Il s'agit en effet de s'assurer une période d'exploitation exclusive (Minon et al., 2015) et que les versions déposées restent des versions " auteur ", ou «preprint » (i.e. précédant le travail de composition, maquettage et rationalisation éditoriale), mettant ainsi en avant l'importance de l'édition Toll Access comme garante de la liberté scientifique et du maintien de la qualité des publications (Minard, 2015; Thompson, 2005). Le problème posé est alors, au niveau économique, celui d'une impossible recherche d'alternative, qui est toujours soumise aux autres impératifs, comme le montrent Joëlle Farchy et Pascal Froissart : « Est-il plus efficace pour diffuser la recherche d'assurer une rente de monopole à Google ou à Elsevier?» (2010: 143), question qui tend d'ailleurs à devenir presque futile lorsqu'on on sait, par exemple, qu'une plate-forme privée de dépôt d'articles en ligne comme Mendeley a été rachetée par Elsevier en 2013.

\section{Le « Freemium » pour résister aux réseaux sociaux privés?}

19 Au-delà des deux roads principalement proposées par les déclarations en faveur du libre accès il est plusieurs autres propositions émanant d'acteurs publics ou de chercheurs engagés dans l'Open Access. En France, la plate-forme d'OpenEdition, portail d'accès à des ressources électroniques en SHS ${ }^{14}$ est à cet égard des plus intéressantes, essayant, par ses actions, de proposer des systèmes permettant d'articuler au mieux les différents impératifs du libre accès. Notamment, entre les deux modèles habituels de financement, la position d'OpenEdition est de promouvoir une «troisième voie platinum ", un " modèle Freemium, dans lequel le texte est en libre accès, mais un ensemble de services à forte valeur ajoutée sont par ailleurs commercialisés ${ }^{15} »$. Il s'agit, ainsi, d'engager la transition vers le libre accès de manière relativement solidaire, en profitant des ressources des institutions prêtes à payer le freemium pour proposer différents services permettant le développement de l'intérêt pour les revues Open Access et leurs articles (métadonnées, données de trafic, de lecture, etc.). En attendant, le modèle semble à même de porter les impératifs du libre accès, notamment en ce qui concerne l'aspect scientifique, ainsi que l'aspect éthico-politique. En revanche, si ce modèle prend bien en considération le fait que c'est l'« intermédiation », voire l'« infomédiation » qui est centrale et susceptible de générer l'essentiel de la valeur ajoutée dans les systèmes de plates-formes d'accès à l'information, il ne propose pas une véritable alternative quant à la question de son financement. Bien au contraire, il prévoit en réalité, lui aussi, un fonctionnement d'agent de type « vectorialiste » (cf. supra). 

principal des contenus scientifiques envisagé par les promoteurs d'OpenEdition. Pour ce qui concerne le cas des universitaires au cœur de l'institution, il faut tempérer l'argument: lorsque les chercheurs produisent un article ou un livre, il ne s'agit pas véritablement de bénévolat, car ce type d'activité entre dans leurs missions à la fois pédagogiques et de recherche, lesquelles sont rémunérées (Dacos, 2012b). La chose est entendue, mais le manque d'investissement de la question à partir d'une critique de l'économie politique tend toutefois à faire oublier que ce modèle d'exploitation du travail scientifique, pour lequel Dacos concède que «les enseignants-chercheurs travaillent plutôt 70 heures [hebdomadaires]"(2012b), repose déjà sur le principe simple d'exploitation du surtravail salarial qui consiste, pour celui qui rémunère la force de travail, à accaparer le travail accompli au-delà du temps de travail effectivement rémunéré (Marx, 1985). Aussi, au-delà de ses avantages évidents et indéniables en termes de facilité d'accès, ce que finit par proposer le modèle freemium consiste à s'engouffrer dans cette exploitation de surtravail scientifique en en renouvelant et en multipliant les formes, et ce, dans le cas le plus enviable où le producteur d'information est effectivement en poste dans une institution de recherche et peut donc inclure sa production au crédit de ses attributions ${ }^{16}$. Ainsi, au lieu de mettre en avant politiquement les particularités du travail scientifique et la nécessité d'en libérer les productions afin d'en faire un potentiel modèle universel de désaliénation du travail, la proposition économique semble consister, a contrario, à en renforcer et diversifier les modalités d'exploitation - au profit des deux autres dimensions du libre accès. Et le fait qu'il puisse s'agir d'exploitation par des infrastructures publiques n'en tempère aucunement la réalité. Cela fait bien longtemps que la critique de l'économie politique analyse l'importance de l'État dans les modalités de mise en place et de reproduction du capitalisme dont il n'est pas la contradiction (Pollock, 1978 ; Mandel, 1976) ${ }^{17}$.

Dans ce cas, il faut reconnaître que la proposition économique d'OpenEdition la conduit à être simple concurrente d'autres plates-formes comme celles dont nous avons parlé supra - même si, à des niveaux autres qu'économique, notamment de qualité technique, de publication scientifique et éditoriale, elle les surclasse complètement. Aussi, la critique de l'économie politique attenante à l'Open Access a du mal à se faire jour dans les moyens mêmes de sa mise en œuvre institutionnelle : on ne change fondamentalement rien à la structure du capitalisme ou à la nécessité de l'exploitation si ce n'est qu'on détermine autrement quels types d'acteurs seront à même d'y tirer leur épingle du jeu. Selon certains commentateurs comme Martin Eve, il est probable que ce soit le point de compromis inhérent au principe même du libre accès: "d'un côté, le libre accès a le potentiel d'éliminer l'impossibilité pour des chercheurs de lire les travaux de leurs pairs. En même temps, toute forme de libre accès selon les définitions institutionnelles [BBB] permettent une valeur d'usage sociétale plus large (et cela inclut son extension aux entités commerciales)» (Eve, 2014 : 67). Sachant que, de surcroît, certaines impulsions étatiques semblent aller vers une « obligation » du libre accès selon les termes présentés, on peut comprendre les réticences de plusieurs commentateurs, voyant alors l'État comme instigateur d'un libre accès renforçant les logiques marchandes. Faut-il pour autant se résoudre à cette seule (absence d')alternative ? Ou chercher, comme le font d'autres auteurs, un dépassement de ce problème dans une quadrature du cercle qui émanerait de nouveaux modèles économiques mieux à même de tenir compte des potentialités économiques nouvelles des biens à l'ère numérique (non rivalité, coût de

Variations, 19 | 2016 
reproduction négligeable, etc. - cf., par exemple, Peugeot, 2011 ; Aigrin, 2005) ? Ce serait peut-être oublier, un peu vite, que le changement social ne vient pas des particularités économiques des biens, fussent-ils numériques, et que «la critique de l'économie est toujours politique » (Garo, $2013: 248)$.

\section{Un Open Access alternatif ?}

22 Ainsi, les quelques critiques que nous avons portées en direction de certaines propositions de financement économique du libre accès ne devraient pour autant pas être prises pour un désaveu du bien-fondé de l'entreprise générale vers la libération de l'accès à l'information scientifique. Persuadés des intérêts scientifiques et éthiques du libre accès, notamment pour les SHS, il nous semble cependant que l'enjeu du libre accès se situe ailleurs. Il tient à sa capacité à préparer un au-delà de l'économie de l'édition scientifique en régime numérique, laquelle appelle une articulation à des propositions vers un au-delà de l'économie capitaliste, selon les propositions de la troisième impulsion qui préside à son émergence. Or, ces problématiques restent par trop éparses dans ce débat qui ne se constitue pas, pour l'heure, sur ce terrain, ce qui limite sa portée politique.

Il nous semble en effet, à l'inverse de certains commentateurs, que le libre accès, en tout cas tel qu'il est exploré en ce moment, que ce soit pratiquement, mais aussi théoriquement, n'a encore rien d'une expérience socialiste qui aurait pénétré le monde scientifique $^{18}$. C'est peut-être ce qui en limite les développements les plus intéressants, ainsi que la cohérence à trouver entre les différentes impulsions. Il est en effet probable que l'inclusion des propositions du libre accès aux agendas des principaux organes de pouvoir tutélaires internationaux du monde scientifique (ministères, advisory board des grandes universités, comités d'organisation des fondations et institutions de financement de la recherche, etc.) en ait réduit les perspectives aux seuls principes à même d'accompagner la libéralisation accrue du secteur de la recherche et de l'enseignement universitaire - et ces principes semblent demeurer les plus essentiels, quels que soient les perspectives politiques des acteurs en présence. Ainsi, au-delà de toutes ses implications, sa défense, comme celle des différentes « routes » (roads) et modalités de sa mise en place, se mesurent en général, que ce soit pour les SHS ou les STM, à l'aune de deux variables : celle de son efficacité dans la diffusion des idées nouvelles auprès des pairs et son incidence sur le référencement bibliométrique (Lawrence, 2001; Antelman, 2004 ; Harnad et al., 2004; Hajjem, Harnad, Gingras, 2005 ; Eysenbach, 2006; Watson, 2009; Gentil-Beccot et al., 2010 ; Hitchcock, 2013) et, plus rarement, son efficacité de diffusion à l'extérieur de la sphère universitaire notamment occidentale (Willinsky, 2006 ; Vecam, 2011; Monnet, 2013; Dacos, 2014 ; Lemercier, 2015) - et en particulier dans le monde des praticiens de la santé des pays du Sud pour le cas de la recherche médicale (Chan et al., 2009). Que l'affirmation euphorique des partisans du libre accès à ce propos doive ou non être tempérée (Craig et al., 2007 montrent ainsi que les scores de citation des articles en Open Access sont peut-être dus à des biais méthodologiques ${ }^{19}$ ), dans les deux cas, c'est à une vision particulièrement utilitariste que nous avons affaire! Et si la recherche d'efficience quant à la diffusion des savoirs théoriques peut, bien évidemment, se justifier tant du point de vue scientifique que du point de vue social ou sanitaire, cette manière de la mesurer, d'inspiration quasi-taylorienne, tend à ne faire du libre accès qu'un instrument d'accompagnement de l'accélération des cadences du travail 
universitaire qui ne gardent plus en tête que l'objectif de résultat final chiffrable, que ce soit pour l'auteur ou les revues. Le débat finit alors par s'orienter vers la recherche de l'intérêt permettant de motiver ou d'inciter les chercheurs à publier dans les revues en libre accès ou concourir au travail d'évaluation de ces revues ${ }^{20}$, ce qui tend à le rabattre sur les termes mêmes qui sont ceux des éditeurs de revues scientifiques non ouvertes et qui leur donnent l'avantage dans un débat qui s'articule autour de l'importance de la rente qu'offre la conservation, dans un catalogue, d'une revue existant de longue date et ayant une réputation bien établie au sein du champ scientifique (Bourdieu, 1997). Même lorsque les arguments à présenter en faveur du libre accès ne s'y limitent pas, notamment dans les débats français sur la question, voire même lorsque cette vision utilitariste peut être par ailleurs critiquée (Dacos, 2014 - cf. infra), il est toutefois fréquent d'y rappeler, quelquefois dans les mêmes textes, qu'une grande partie des revues de SHS a « rejoint un portail, principalement Cairn.info et Revues.org, qui regroupent chacun quelque 400 revues, dont environ 200 classées par l'AERES » (Lemercier, 2015 : 54, nous soulignons). Bien sûr, les revues, et notamment les revues «fermées" (Toll Access) jouant toujours un rôle important dans le monde académique, "en se repliant quasi exclusivement sur la fonction de validation et certification de la publication" (Mounier, 2010a: 26), on comprend aisément la nécessité d'apporter des preuves qu'une vision plus ouverte de la recherche a d'ores et déjà pris un poids scientifique important, selon les termes mêmes des discours dominant le débat au moment même où l'on décide de l'investir. Mais croire que celui-ci n'en sera pas davantage à même d'opérer une réification de l'utilité sociale de l'information scientifique (sa "valeur d'usage ») versant en bibliométrie, libéralisation et contrôle accru du travail scientifique (selon les termes de la "valeur d'échange ») ${ }^{21}$ est probablement illusoire, surtout s'il n'est pas de proposition politique et sociale radicale forçant un autre mouvement. Les arguments pour l'Open Access rejoignent alors les arguments contre l'Open Access : il s'agit de trouver le one best way permettant de maintenir un "haut niveau de la recherche ", ainsi que les réputations (des chercheurs, de leurs institutions et des revues) et, in fine, l'argument ultime n'est qu'affaire de choix opératoire, éventuellement au cas par cas. Aussi n'est-il pas rare de voir s'affoler les directeurs de publication des revues classées qui, s'ils peuvent entendre les avantages du libre accès, s'inquiètent à juste titre de la possibilité économique du maintien de leur revue dans la période de transition (Anheim, 2015).

Il faut, ici, remarquer que les règles mêmes du débat sont déjà au service d'une vision néo-libérale de la recherche. Les routines et habitudes, forgées à l'aune de décennies d'une économie de monopoles de l'édition scientifique, qui a maintenu la croyance que ces monopoles étaient la seule possibilité pour préserver une édition scientifique de qualité, ont tendance à présenter toute tentative qui en sortirait comme pure "utopie ", voire pure "idéologie " qui n'aurait pas sa place dès lors qu'on cherche à réfléchir sérieusement l'économie des revues ("il faut répondre à la question "qui va payer ?" »: Wolton, 2010 : 15 - Minard, 2015 ; Minon et al., 2015). Des arguments fréquents de type "pourquoi vouloir distribuer gratuitement des revues électroniques que personne n'a songé à distribuer gratuitement lorsqu'elles étaient uniquement sur papier ?22 ", qui insistent en général sur l'importance des revues «Toll Access » et des barrières mobiles longues, garantes de la qualité de la production scientifique (assumant quelquefois de réserver cette dernière à la seule population des enseignants-chercheurs et étudiants Minard, 2015 : 15) nous paraissent aussi irrecevables que la proposition selon laquelle le libre accès doit être défendu en termes de «biblio-diversité » (Lemercier, 2015), dans une 
perspective de dissémination de la recherche qui tend à se limiter parfois à un simple projet de vulgarisation (Dacos, 2014).

Ce dernier argument oublie, en effet, comme nous l'avons vu, que les modalités d'accès aux contenus scientifiques doivent être intégrées à une réflexion d'ensemble sur l'économie de l'intermédiation et ses conséquences sur le déplacement, en son sein, des modalités de l'exploitation. Il doit également, quel que soit le nom qu'on leur donne, se coupler à une critique des nouveaux indicateurs "webométriques» qui recréent des mesures du même type que le "facteur d'impact» que les tenants de l'open Access s'accordent pourtant à déqualifier (Dacos, 2014 ; Campbell, 2008). La logique devient alors quasiment celle d'une gestion du trafic numérique, qu'on cherche à exacerber par des moyens qui finissent par ne même plus satisfaire les intentions scientifiques du libre accès. Ainsi, lorsque Dacos (2014) appelle les SHS à " accepter de devenir attractives ", à "s'adresser au citoyen ", montrant que grâce à certains outils développés par OpenEdition pour «s'adresser au public», et d'après le trafic enregistré par ces outils (nombre de vues), on peut imaginer que des lecteurs hors du cercle académique ont pu accéder aux travaux scientifiques. De tels arguments laissent entendre une réalisation de ce qu'analysent Farchy et Froissart à propos du moteur de recherche spécialisé Google Scholar: "Dans cette économie de l'audience et du trafic, on finit par oublier l'information, le contenu lui-même, au profit d'une seule réflexion sur la gestion des flux et le contrôle des interfaces par lesquelles l'utilisateur entre sur le réseau » (2010: 143). Mais pour autant, espérer, en suivant le premier argument, que nier le libre accès suffit pour se prémunir contre ses travers, revient à ne pas voir que les mutations numériques en ont accompagné d'autres, économiques et sociales. Celles-ci ont pourtant opéré un certain nombre de changements majeurs au sein de l'université, pour lesquels une grille de lecture issue des grandes heures de l'édition papier ne convient guère, et ce, pas uniquement au niveau des seules problématiques qui touchent à l'édition scientifique elle-même. Ainsi, outre les changements concernant les nouveaux modèles de l'économie électronique, la période numérique coïncide aussi, dans une large partie des pays du Nord, avec un ensemble de crises liées au travail, condamnant au chômage, parfois de longue durée, une génération se prévalant pourtant d'un niveau de certification scolaire des plus importants ${ }^{23}$. Et si les changements dans le monde du travail ont permis des situations telles que «lors des troisième et quatrième trimestres de 2010 , les sociétés américaines ont affiché des profits record au moment même où le US Bureau of Labor and Statistics a rendu compte d'un taux de chômage réel à 17 \% » (Ross, $2013: 16$ ), l'Université n'a pas été épargnée. Aux États-Unis comme en Europe et notamment depuis 2008-2009, elle a connu des stagnations, voire des réductions de ses effectifs d'enseignantschercheurs titulaires, alors que les populations étudiantes ne cessent, elles, d'augmenter (cf. Miquelard-Garnier, 2015 pour le cas français ${ }^{24}$ ). Vouloir ainsi maintenir le système des revues et de leur prestige selon le modèle traditionnel, c'est déjà encourager au niveau de l'offre, le goulet d'étranglement et une concurrence accrue des chercheurs sur les titres de revue les plus prestigieux et leur « facteur d'impact » supposé - qui est aujourd'hui la monnaie d'échange principale du capital scientifique (Bourdieu, 1997) accompagnant la libéralisation de l'enseignement supérieur. C'est donner à ces titres et à leurs comités éditoriaux, par le simple fait qu'ils soient déjà là, un pouvoir délégataire sur les qualifications et les recrutements (Eve, 2014:14; Smith, 2013), pouvoir qui ne se justifie qu'assez rarement du point de vue scientifique par rapport à d'autres publications scientifiques, peut-être arrivées plus tard (notamment les revues en Open Access - Suber, 2010) ${ }^{25}$. Bref, c'est finalement, là encore, vouloir maintenir à tout prix des rentes de 
situation qui ne s'expriment que selon les termes instrumentaux et quantitatifs de la valeur d'échange (ici d'ordre réputationnel). Ensuite, du côté de la demande, défendre qu'il convient de réserver l'accès et/ou la participation à la publication scientifique aux seuls titulaires d'un poste ou d'une position d'étudiant dans une institution de recherche signifie donc qu'on assume de former des étudiants par la recherche pour... les en couper à la fin de leurs études (puisqu'une grande partie d'entre eux ne pourra embrasser une carrière universitaire). Cette orientation limite la portée sociale de la recherche universitaire et surtout conduit à abandonner, un peu vite, la possibilité qu'une formation par la recherche (y compris pour des publics ne se destinant pour autant pas aux métiers de la recherche) puisse avoir droit de cité à l'heure d'une injonction constante à la "professionnalisation » dans le monde universitaire. Car l'apprentissage par la recherche doit ensuite s'actualiser dans une fréquentation assidue, voire une participation à la publication. Certains opposants à l'open Access en font d'ailleurs quelquefois un argument, à l'instar de Robin Osborne, pour qui la recherche académique n'est pas compatible avec un principe d'accès libre et gratuit pour la simple raison qu'elle ne consiste pas en une simple "base de données» de résultats, mais qu'il s'agit d'un «processus » qui doit faire l'objet d'un apprentissage, apprentissage que les universités enseignent contre rémunération : "Pour ceux qui veulent avoir accès, il y a un coût d'admission : ils doivent investir dans les prérequis éducationnels pour leur permettre de comprendre le langage utilisé. Les pratiques de publication actuelles font en sorte que ce seuil d'entrée pour comprendre mon langage soit le moins élevé possible. L'Open Access élèvera ce seuil. Bien plus de travaux seront téléchargés, bien moins seront compris » (Osborne, 2013).

26 Si le fond de l'argument peut être entendu, il nous semble qu'il faut plutôt en tirer la conclusion inverse et viser une libération de l'accès à l'enseignement supérieur (pour laquelle il faut exiger que les États se donnent des moyens accrus, notamment humains, en encourageant la création de postes stables des personnels enseignants, chercheurs, administratifs et techniques), permettant une libération du travail de ces personnels par une réduction des tâches et du temps de travail. Celle-ci irait ainsi de concert avec une libération de l'accès aux publications scientifiques, dont il faudrait alors veiller à ce qu'elle soit bien une conséquence de la libération de l'accès à l'enseignement supérieur et du travail. Pour ce qui concerne la France, il ne s'agit donc pas de « rend[re] par avance les armes devant les cadres de Bercy qui croient qu'Internet dispense de tout effort, comme leurs prédécesseurs des années 1980 ont, en découvrant les machines à traitement de texte, cru bon de supprimer nombre de postes de personnels administratifs et techniques dans l'enseignement supérieur » (Fridenson, 2015 : 98), mais de lutter pour maintenir ces postes, afin de ne pas faire reposer toute la charge administrative ou technique sur les personnels restants voire, comme nous l'avons souligné supra, de profiter du «travail gratuit » de jeunes chercheurs en formation ou de chercheurs précaires en marge des universités. C'est de cette situation qu'est né le mouvement américain des alternative academics (" alt-ac jobs») dans les humanités numériques (Berra, 2012 ; Granjon, Magis dans ce numéro); il faut l'intégrer à une vision plus large, qui peut aussi s'articuler autour des problématiques du libre accès et qui doit se constituer politiquement. En revanche, il convient de considérer que toutes les tâches d'édition et de publication scientifiques (représentant un véritable travail qu'il ne s'agit aucunement de minimiser) entrent dans les missions des personnels recrutés et n'a plus besoin de ressources supplémentaires pour revenir au public, selon un principe cher aux sciences sociales critiques, de rendre aux acteurs le savoir construit à partir d'une analyse de leurs expériences (Granjon, 2015). Les 
fonds alloués aux bibliothèques pour le paiement des abonnements aux catalogues Toll Access seraient ainsi redistribuables non pas, comme proposé par certains commentateurs, vers un financement général d'un modèle auteur-payeur (développant la "Gold road ») plutôt que lecteur-payeur (Schimmer et al., 2015), mais plutôt en direction de l'embauche de tous ces personnels. En d'autres termes, il s'agit de revenir aux propositions critiques dont nous parlions au début, en leur donnant une impulsion pratique sur le plan politique: partir du principe que le travail des enseignantschercheurs est potentiellement libérateur, et qu'il faut maintenir ce caractère en limitant les interférences des multiples tâches administratives avec ses missions principales et en permettant sa réalisation dans des conditions convenables (en réduisant les effectifs d'étudiants par classe, de doctorants par professeur, etc.). Il convient ensuite d'en faire un modèle de libération des autres tâches, par une réduction générale et drastique du temps de travail, permettant de libérer l'expression des potentialités créatives de tous les autres individus - parmi lesquelles, celles de se documenter à l'information scientifique, produisant un ensemble potentiel d'unexpected readers. Et dans l'optique d'un développement honnête de l'Open Access, dépassant sa simple visée de récupération du «savoir » par le monde industriel dans une économie basée sur l'innovation, il faut bien voir que l'impulsion «éthico-politique » du libre accès ne pourra se déployer que dans une société ainsi libérée. En effet, tant que cette libération générale de l'accès à l'enseignement supérieur comme du travail ne sera pas atteinte, il ne suffira pas que l'accès aux ressources soit libre et techniquement assuré pour que les compétences permettant aux «lecteurs inattendus » de les consulter se développent d'elles-mêmes. Comme pour le cas de la soi-disant «fracture numérique » (Granjon, 2011), il ne suffit pas de considérer que le problème de l'accès et de la dissémination sociale de la science se limite ici à une simple question technique ou à son financement : la libération véritable de l'accès aux ressources scientifiques ne peut se réaliser que dans le cadre d'une libération sociale plus large.

Dès lors, il faut être vigilant afin que l'argument évite de se polariser entre: (1) des visions conservatrices souhaitant limiter les abus des nouveaux monopoles des plateformes du web sur les travaux de la recherche scientifique, mais pour maintenir la réservation de la production et de la diffusion de l'information scientifique à des petites sociétés autorisées et égocentrées d'auteurs, lecteurs et éditeurs qui n'est plus en adéquation avec la réalité tant technique que sociale ou économique de l'époque ; (2) des visions prospectives, parfois technodéterministes, qui cherchent à faire découler les nécessités politiques et économiques des possibilités techniques oubliant (ou pas) que ces dernières peuvent tout à fait, hors de toute réflexion proprement politique préalable, être employées à des fins de contrôle de l'activité scientifique par de très gros acteurs de l'internet et les États. La question n'est évidemment pas de faire "table rase du passé ", contrairement à ce que dénoncent plusieurs contradicteurs de l'open Access, mais d'admettre que « les tentatives de résistance à la valorisation [en termes économiques] de l'enseignement supérieur par la recherche d'une restauration d'une configuration précédente - lorsque l'université n'était pas complètement perçue comme un moteur de croissance - sont malavisées » (Winn, 2013). Il convient alors plutôt d'analyser le passé et de savoir y lire et penser les imbrications dans les politiques de la recherche scientifique, entre aspects scientifiques, politiques et économiques, au-delà de la tour d'ivoire universitaire, pour ne plus prendre un élément pour un autre et réorienter la hiérarchie des priorités. En l'occurrence, la préséance devrait être d'ordre politique et pourrait se ressourcer dans une critique élargie, d'inspiration marxiste, de l'économie politique (qui 
n'oublie pas que toute critique économique doit sourdre d'une critique sociale), laquelle fait l'objet d'un regain d'intérêt, notamment depuis la crise de 2008 (Fuchs, Mosco, 2012). delà, on constate que l'essentiel des discussions, qu'elles soient pour ou contre, tendent finalement à passer à côté de ses potentialités critiques les plus radicales. Bien sûr, ce ne sont pas vraiment ces possibles que les principales impulsions en sa direction ont visé : nous avons bien vu la prégnance de deux impulsions, que nous avons appelé "scientifique » et "éthico-politique », principalement centrées sur l'accroissement de l'efficacité de la diffusion scientifique, à l'intérieur comme à l'extérieur du secteur universitaire. Mais une telle relégation de ces ferments plus critiques et de leur intégration partielle à un ethos libriste, peut paraître étrange, alors même que les mutations actuelles de l'enseignement supérieur, desquelles l'open Access fait partie intégrante, répondent dangereusement aux injonctions des principales mutations néolibérales du capitalisme ${ }^{26}$. La problématique de l'Open Access, déjà réintégrée aux nouveaux modèles économiques du capitalisme "vectorialiste" (Wark, 2006; Citton, 2013) et leurs nouveaux modes d'exploitation du digital labour (Scholz, 2013), comme aux nouvelles modalités institutionnelles d'exploitation du travail scientifique, voit se réduire comme peau de chagrin les propositions radicales qu'elle pourrait pourtant faire éclore. Il faudrait pour cela y prêter une attention véritable et ne croire, ni que ces potentialités pourraient se déployer d'elles-mêmes, ni envisager le fait qu'elles ne se soient pas déjà pleinement déployées comme la preuve qu'elles seraient à remiser au rang des dernières utopies naïves charriées par le numérique. Sur ce principe, il est d'ailleurs probable que notre proposition ne reçoive, à son tour, qu'une disqualification immédiate pour « utopie » voire « idéologie », ne s'appuyant sur aucune véritable démonstration d'ordre (biblioth)économique afin d'apporter les preuves de sa viabilité. Mais quitte à chercher des solutions économiques - ce à quoi tous les acteurs en présence, enthousiastes comme réfractaires au libre accès, semblent en réalité occupés -, rappelant que «le modèle économique reste à inventer » (Monnet, 2013), autant que ces solutions servent à soutenir et accompagner une mutation véritablement radicale, dépassant de fait le débat scientifique, selon une perspective centrale des sciences sociales critiques (Granjon, 2015). Cela ne pourra cependant advenir qu'à la condition d'une prise de conscience des universitaires sur le fonctionnement des activités auxquelles ils participent, notamment au niveau économique. Parce que si, dans les discours autour du libre accès, il est un élément qui ne fait pas débat, c'est bien le manque d'intérêt des chercheurs pour les fonctionnements économiques des revues dans lesquelles ils publient et qui sont au centre de la valorisation de leur activité.

C'est ainsi, là, un des principaux enjeux des débats actuels sur l'Open Access et celui pour lequel il nous a semblé nécessaire de produire cette réflexion : l'urgence d'une prise de conscience des chercheurs, notamment critiques, quant aux véritables implications économiques liées à leurs activités. Ce serait tout de même un point de départ nécessaire, dans l'optique d'une recherche de solutions alternatives - à condition de ne pas oublier que chaque «solution » économique, charrie avec elle un modèle de ce que doit être la science et, in fine, la société. Il est donc important que les chercheurs, en particulier critiques, se mettent à investir ce débat et cherchent à revivifier les ferments les plus 
radicaux du libre accès, de manière à expérimenter certaines de leurs propositions dans le cadre d'une praxis concrète, autour d'activités de production qui les concernent au premier chef. Prima caritas incipit a seipso.

\section{BIBLIOGRAPHIE}

Aigrin (Philippe), « De l'accès libre à la science ouverte », in Vecam (ed.), Libres savoirs. Les biens communs de la connaissance, Caen, C\&F, 2011, pp. 77-85.

Aigrin (Philippe), Cause commune. L'information entre bien commun et propriété, Paris, Fayard, 2005.

Anheim (Étienne), «Le numérique et l'économie éditoriale des revues scientifiques », Revue d'histoire moderne et contemporaine, $\mathrm{n}^{\circ} 62-4$ bis, 2015, pp. 22-32.

Antelman (Kristin), « Do Open-Access Articles Have a Greater Research Impact? », College \& Research Libraries, vol. $65 \mathrm{n}^{\circ}$ 5, 2004, pp. 372-382.

Apt (Krzysztof R.), « One More Revolution to Make: Free Scientific Publishing », Communications of the ACM, vol. $44 \mathrm{n}^{\circ}$ 5, 2001, pp. 25-28.

Beall (Jeffrey), « The Open-Access Movement is Not Really about Open Access ", tripleC: Communication, Capitalism \& Critique, vol. 11 n 2, 2013, pp. 589-597.

Bergstrom (Thedore C.), « Free Labor for Costly Journals? », Journal of Economic Perspectives, vol. 15 $\mathrm{n}^{\circ} 4,2001$, pp. 183-198.

Berra (Aurélien), « Faire des humanités numériques », in Mounier (Pierre) dir., Read/Write Book 2. Une introduction aux humanités numériques, Marseille, OpenEdition Press, 2012, http:// books.openedition.org/oep/238.

Bouquillion (Philippe), Matthews (Jacob T.), Le web collaboratif, Grenoble, PUG, 2010.

Bourdieu (Pierre), Les usages sociaux de la science. Pour une sociologie clinique du champ scientifique, Paris, INRA Éditions, 1997.

Broca (Sébastien), Utopie du logiciel libre. Du bricolage informatique à la réinvention sociale, Paris, Le Passager Clandestin, 2013.

Case (Mary M.), « Measuring Cost Effectiveness of Journals: the Wisconcin Experience », ARL Newsletter, 1999.

Chan (Leslie), Arunachalam (Subbiah), Kirsop (Barbara), « The chain of communication in health science: from researcher to health worker through open access », Open Medicine, vol. $3 \mathrm{n}^{\circ} 3$, pp. 111-119, http://www.ncbi.nlm.nih.gov/pmc/articles/PMC2765774/.

Chantepie (Philippe), Le Diberder (Alain), Révolutions numériques et industries culturelles, Paris, La Découverte, 2010.

Chartron (Ghislaine), «Stratégie, politique et reformulation de l'open access », Revue française des sciences de l'information et de la communication, $\mathrm{n}^{\circ} 8$, http://rfsic.revues.org/1836.

Citton (Yves), « Humanités numériques. Une médiapolitique des savoirs encore à inventer », Multitudes, vol. 2 n59, 2015, pp. 169-180. 
Citton (Yves), «Économie de l'attention et nouvelles exploitations numériques », Multitudes, vol. $3 n^{\circ} 54,2013$, pp. 163-175.

Craig (Ian), Plume (Andrew M.), McVeigh (Marie), Pringle (James), Amin (Mayur), « Do Open Access Articles Have a Greater Citation Impact? A Critial View of the Litterature », Journal of Infometrics, vol. $1 \mathrm{n}^{\circ}$ 3, 2007, pp. 239-248.

Dacos (Marin), « Un engagement de diamant pour l'open access - Position du Comité des sciences sociales de Science Europe », Blogo-Numericus, 18 aout 2015, http://bn.hypotheses.org/11642.

Dacos (Marin), « Bibliodiversité et accès ouvert. Les enjeux des infrastructures numériques d'édition électronique ouverte en sciences humaines et sociales ", Blogo-numericus, 2014, http:// bn.hypotheses.org/11585\#_ftn11.

Dacos (Marin), « Vers des médias numériques en sciences humaines et sociales : une contribution à l'épanouissement de la place des sciences humaines et sociales dans les sociétés contemporaines », Tracés, $\mathrm{n}^{\circ}$ hors-série, 2012a, pp. 205-223, http://traces.revues.org/5534.

Dacos (Marin), « Gratuité ou libre accès ? Poser les termes du débat, c'est déjà y répondre en partie », Blogo-Numericus, 28 oct. 2012b, http://bn.hypotheses.org/11070.

Dewatripont (Mathias), Ginsburgh (Victor), Legros (Patrick), Walckiers (Alexis), « Pricing of scientific journals and market power », Journal of European Economic Association, vol. 5 n 2-3, 2007, pp. 400-410.

Eve (Martin P.), Open Access and the Humanities. Contexts, Controversies and the Future, Cambridge, Cambridge University Press, 2014.

Eysenbach (Gunther), « Citation advantage of open access articles », PLoS Biol, vol. $4 \mathrm{n}^{\circ}$ 5, 2006, pp. 692-698.

Farchy (Joëlle), Froissart (Pascal), Méadel (Cécile), « Introduction », Hermes, n 57, 2010, pp. 9-12.

Farchy (Joëlle), Froissart (Pascal), « Le marché de l'édition scientifique entre accès "propriétaire” et accès "libre" », Hermes, n 57, 2010, pp. 137-150.

Fridenson (Patrick), « En France, au cœur de la révolution numérique internationale », Revue d'histoire moderne et contemporaine, $\mathrm{n}^{\circ}$ 62-4 bis, 2015, pp. 83-99.

Fuchs (Christian), Digital labour and Karl Marx, New York/Londres, Routledge, 2014a.

Fuchs (Christian), « Critique of the Political Economy of Informational Capitalism and Social Media ", in Fuchs (Christian), Sandoval (Marisol) eds., Critique, Social Media and the Information Society, New York/Londres, Routledge, 2014b.

Fuchs (Christian), Mosco (Vincent) ed., « Marx is back. The importance of Marxist theory and research for Critical Communication Studies Today, triplec: Communication, Capitalism \& Critique, vol. $10 \mathrm{n}^{\circ}$ 2, pp. 127-632.

Garo (Isabelle), L'Or des images. Art-Monnaie-Capital, Montreuil, La Ville Brûle, 2013.

Gentil-Beccot (Anne), Mele (Salvatore), Brooks (Travis), « Citing and Reading Behaviours in HighEnergy Physics », Scientometrics, vol. 84 n², 2010, pp. 345-355.

Ginsparg (Paul), «First Steps Towars Electronic Research Communication », Computers in Physics, vol. $8 \mathrm{n}^{\circ} 4,1994$, pp. 390-396.

Granger (Christophe), La destruction de l'université française, Paris, La Fabrique, 2015.

Granjon (Fabien), « Du matérialisme comme principium d'un agenda de la recherche critique en communication », Questions de communication, $\mathrm{n}^{\circ}$ 28, 2015, pp. 157-190. 
Granjon (Fabien), « Fracture numérique. », Communications, n 88 vol. 1, 2011, pp. 67-74. Gusterson (Hugh), « Want to Change Academic Publishing? Just Say No », The Chronicle of Higher Education, 23 sept. 2012, http://chronicle.com/article/Want-to-Change-Academic/134546? cid=trend_right_a.

Hajjem (Chawki), Harnad (Stevan), Gingras (Yves), « Ten-Year Cross-Disciplinary Comparison of the Growth of Open Access and How it Increases Research Citation Impact », IEEE Data Engineering Bulletin, vol $28 \mathrm{n}^{\circ}$ 4, 2005, pp. 39-47, http://eprints.soton.ac.uk/262906/.

Harnad (Stevan), « The Green Road to Open Access: A Leveraged Transition », in Gacs (Anna) ed., The Culture of Periodicals from the Perspective of the Electronic Age, Paris, L'Harmattan, 2007, pp. 99-106, http://eprints.soton.ac.uk/265753/1/greenroad.html.

Harnad (Stevan), « Post-Gutenberg Galaxy: The Fourth Revolution in the Means of Production of Knowledge », Public-Access Computer Systems Review, vol. 2 n 1, 1991, pp. 39-53, http:// eprints.soton.ac.uk/253376/2/harnad91.postgutenberg.html.

Harnad (Stevan), Brody (Tim), Vallieres (François), Carr (Les), Hitchcock (Steve), Gingras (Yves), Oppenheim (Charles), Stamerjohanns (Heinrich) \& Hilf (Eberhard), « The Access/Impact Problem and the Green and Gold Roads to Open Access ", Serials Review, vol. 30 n 4, 2004, http:// users.ecs.soton.ac.uk/harnad/Temp/impact.html.

Hesmondhalgh (David), « User-generated content, free labour and the cultural industries. », Ephemera, vol. 10, $\mathrm{n}^{\circ}$ 3/4, 2010, pp. 267-284.

Hitchcock (Steve), « The Effect of Open Access and Downloads ("hits") on Citation Impact: A Bibliography of Studies », Southampton, University of Southampton, 2013, http:// eprints.soton.ac.uk/354006/.

Krichel (Thomas), « Working towards an Open Library for Economics: the RePEc Project », 2000, http://openlib.org/home/krichel/myers.html.

Kyrillidou (Martha), « Spending More for Less », ARL Report, juin 1999, https:// www.arlstatistics.org/publications.

Lawrence (Steve), « Free online availability substantially increases a paper's impact », Nature, 411, 2001, pp. 521.

Lemercier (Claire), « Pour qui écrivons-nous? », Revue d'histoire moderne et contemporaine, $\mathrm{n}^{\circ}$ 62-4 bis, 2015, pp. 43-61.

Marx (Karl), Le Capital. Livre I - section I-IV, Paris, Flammarion, 1985.

Mele (Salvatore), « Open Access publishing in high-energy physics », OCLC Systems \& Services, vol. $25, n^{\circ} 1,2009$, pp. 20-34.

Mele (Salvatore), « Le projet SCOAP3, une révolution en physique des hautes énergies », Hermes, $\mathrm{n}$ -57, 2010, pp. 161-163.

Minard (Philippe), « Les revues à l'âge numérique : au péril de l'idéologie », Revue d'histoire moderne et contemporaine, $\mathrm{n}^{\circ}$ 62-4 bis, 2015, pp. 8-21.

Minon (Marc), Parisot (Thomas), Bureau (Stéphane), « Les revues SHS de langue française à la croisée des chemins ", Revue d'histoire moderne et contemporaine, ${ }^{\circ}$ 62-4 bis, 2015, pp. 71-82.

Monnet (Éric), « Open access: la schizophrénie française », Le carnet de Tracés, 2013, http:// traces.hypotheses.org/805.

Mounier (Pierre), « Le libre accès : entre idéal et nécessité », Hermes, n 57, 2010a, pp. 23-30. 
Mounier (Pierre), «L'édition électronique : un nouvel eldorado pour les sciences humaines ? », in Dacos (Marin) dir., Read/Write Book. Le livre inscriptible, Marseille, OpenEdition Press, 2010b, http://books.openedition.org/oep/169.

Osborne (Robin), « Why Open Access Makes No Sense », The Guardian, 8 juin 2013, http:// www.theguardian.com/higher-education-network/blog/2013/jul/08/open-access-makes-nosense.

Ostrom (Elinor), Hess (Catherine) dir., Understanding Knowledge as Commons, Cambridge MA, MIT Press, 2007.

Peugeot (Valérie), «Les biens communs, une utopie pragmatique », in Vecam (ed.), Libres savoirs. Les biens communs de la connaissance, Caen, C\&F, 2011.

Pollock (Friedrich), « State Capitalism: Its Possibilities and Limitations », Studies in Philosophy and Social Science, $\mathrm{n}^{\circ}$ 9, pp. 200-225.

Ross (Andrew), «In Search of the Lost Paycheck », in Scholz (Trebor) ed., Digital Labor. The Internet as Playground and Factory, New-York, Routledge, 2013.

Sample (Ian), « Harvard University says it can't afford journal publisher's prices », The Guardian, 24 avril 2012, https://www.theguardian.com/science/2012/apr/24/harvard-university-journalpublishers-prices.

Schimmer (Ralf), Geschuhn (Kai Karin), Vogler (Andreas), « Disrupting the subscription journals' business model for the necessary large-scale transformation to open access », 2015, http:// hdl.handle.net/11858/00-001M-0000-0026-C274-7.

Scholz (Trebor) dir., Digital Labor. The Internet as Playground and Factory, New-York, Routledge, 2013.

Smith (Richard), « The Irrationality of the REF », BMJ, 7 mai 2013, http://blogs.bmj.com/ bmj/2013/05/07/richard-smith-the-irrationality-of-the-ref/.

Smith (Kevin), « The unexpected reader », Scholarly Communications @ Duke, 15 novembre 2011, http://blogs.library.duke.edu/scholcomm/2011/11/15/the-unexpected-reader/.

Suber (Peter), Open Access, Cambridge MA, MIT Press, 2012.

Suber (Peter), « Thoughts on prestige, quality, and open access », Logos, vol. $21 \mathrm{n}^{\circ} 1-2,2010$, pp. 115-128.

Terranova (Tiziana), Network Cultures. Politics for the Information Age, London, Pluto Press, 2004.

Thompson (John B.), Books in the Digital Age: The Transformation of Academic and Higher Education Publishing in Britain and the United States, Cambridge MA, Polity Press, 2005.

Vecam (ed.), Libres savoirs. Les biens communs de la connaissance, Caen, C\&F, 2011.

Vecam (ed.), Pouvoir Savoir. Le développement face aux biens communs de l'information et à la propriété intellectuelle, Caen, C\&F, 2005.

Vest (Charles M.), « Openness and Globalization in Higher Education: The Age of the Internet, Terrorism and Opportunity », Center for Studies in Higher Education, University of Berkeley, juil. 2006, http://www.cshe.berkeley.edu/sites/default/files/shared/publications/docs/ ROP.Vest.Openness.7.06.pdf.

Wark (McKenzie), Un manifeste hacker, Paris, CriticalSecret, 2006.

Willinsky (John), The Access Principle: The Case for Open Access to Research and Scholarship, Cambridge MA, MIT Press, 2006. 
Winn (Joss), « Helplessness », Joss Winn, 1 juillet 2013, http://josswinn.org/2013/07/

helplessness/.

Wolton (Dominique), « Abondance et gratuité : pourquoi faire et jusqu'où ? », Hermes, n 57, 2010, pp. 13-19.

\section{NOTES DE FIN}

1. Cf. "Pour une science ouverte à tous" (Le Monde, 7 mars 2016), tribune signée par une trentaine de chercheurs: http://www.lemonde.fr/sciences/article/2016/03/07/pour-unescience-ouverte-a-tous_4878011_1650684.html. Fin mars 2016, une pétition portant le même titre a été déposée sur http://change.org, afin d'encourager les sénateurs à voter la loi et notamment ses articles les plus engagés en faveur de l'ouverture de l'accès aux données scientifiques. Un autre collectif de chercheurs a signé, pour sa part, une tribune titrée "Les revues de sciences sociales en danger » (Libération, 17 mars 2016) : http://www.liberation.fr/debats/2016/03/17/lesrevues-de-sciences-sociales-en-danger_1440203. Nota bene: sauf indication contraire, tous les hyperliens ont été vérifiés le 30 mars 2016.

2. Dans le cadre du présent article, nous n'entrerons pas dans le débat à ce niveau de raffinement et utiliserons les trois termes comme synonymes.

3. Cette crise, à laquelle nous reviendrons, a fait suite à une concentration continue de l'édition scientifique et sa commercialisation par «bouquets " par de très gros éditeurs, à des prix ayant augmenté bien plus vite que l'inflation depuis les années 1960 (Dewatripont et al., 2007), et surtout bien plus vite que les budget des bibliothèques ou organismes de recherche susceptibles de s'y abonner (Bergstrom, 2001).

4. http://arxiv.org/.

5. http://eprints.soton.ac.uk/252626/1/index.html.

6. http://www.nap.edu/readingroom.php?book=exch\&page=summary.html.

7. Notamment sur des thématiques spécifiques comme la diffusion des traitements antirétroviraux ou l'autorisation de commercialisation des graines OGM. On pourra consulter les ouvrages édités par l'association Vecam $(2005,2011)$ pour un aperçu.

8. Chiffres de l'Association of Research Libraries, Washington, DC : http://www.arl.org/storage/ documents/expenditure-trends.pdf.

9. Reed Elsevier, jusqu'en février 2015. Principal éditeur en ligne de mire des critiques, le chiffre d'affaires total du groupe pour l'année 2014 s'élève à 5773 millions de livres sterling (plus de sept milliards trois cent millions d'euros - taux de conversion moyen de décembre 2014 ; données INSEE), auquel la division consacrée à l'édition scientifique, technique et médicale a contribué à la hauteur de 2048 millions de livres (plus de deux milliards et demi d'euros) : http:// www.relx.com/mediacentre/pressreleases/2015/Documents/reed-elsevier-results-2014pressrelease.pdf.

10. Cette stratégie a d'abord été initiée par Public Library of Science (PLOS): http:// www.plos.org.

11. En France, l'archive ouverte nationale, HAL (Hyper Articles en Ligne) est développée depuis 2001 : https://hal.archives-ouvertes.fr/.

12. http://www.agence-nationale-recherche.fr/informations/actualites/detail/l-anr-incite-leschercheurs-a-integrer-leurs-publications-dans-le-systeme-d-archives-ouvertes/.

13. Figure mythique $d u$ « lecteur inattendu » des humanités numériques, dans le texte original de Kevin Smith (2011), il s'agit d'une jeune élève de lycée de quinze ans qui, en lisant des articles spécialisés en biologie via des accès « test » à des catalogues scientifiques, aurait fini par écrire un 
article à son tour et pour le proposer au rédacteur en chef de la revue PLoS Computational Biology. Ce dernier, attestant de l'innovation majeure proposée par l'article l'a finalement publié, non sans l'avoir même préalablement proposé à la prestigieuse revue Science.

14. OpenEdition participe du projet développé par le Centre pour L'édition Électronique Ouverte (Cléo) dirigé par Marin Dacos et Pierre Mounier, lequel propose également une base de revues, un calendrier et un blog agrégeant plusieurs carnets de recherches.

15. http://leo.hypotheses.org/9953.

16. Il faut, en effet, garder à l'esprit que nombre des producteurs de publications scientifiques sont aussi des précaires de l'université (étudiants non-financés, docteurs non embauchés...) qui peuvent ne bénéficier d'aucune forme de rétribution pécuniaire pour ce type de travail.

17. Charlotte Hess et Elinor Ostrom, appuient d'ailleurs à ce propos que les universités (américaines) se trouvent " des deux cotés du débat des communs, augmentant le dépôt des brevets en dépendant de plus en plus sur les crédits privés pour la recherche tout en encourageant en même temps le libre accès et mettant à disposition des espaces numériques de dépôt pour les productions de leurs enseignants-chercheurs » (2007:10).

18. Cf. https://molphil.wordpress.com/2009/02/27/socialism-in-science-or-why-open-accessmay-ultimately-fail/ et les trois articles qui y font suite, cf. également l'article de Mike P. Taylor : http://svpow.com/2013/06/11/why-open-access-is-not-socialism/ . Si cette rhétorique d'épouvantail tendant à accuser l'Open Access de démarche socialiste (ce dernier terme résonnant alors comme une sentence maccarthyste) est répandue dans les articles émanant de chercheurs (non-critiques), on pourra même en trouver la trace jusque dans un article rédigé par un directeur de bibliothèque universitaire du Colorado, Jeffrey Beall : « une analyse des discours des partisans de l'Open Access révèle que le véritable but du mouvement est d'éliminer les éditeurs à but lucratif et faire de l'édition académique une entreprise coopérative et socialiste » (2013:590). 19. Il est ici tout de même à noter que les auteurs de ce texte sont tous employés de grandes majors de l'édition scientifique (Elsevier, Thomson Scientific et Wiley-Blackwell).

20. Cf. https://molphil.wordpress.com/2009/02/27/socialism-in-science-or-why-open-accessmay-ultimately-fail/

21. Et ce, malgré les appels à des visions moins quantitatives de l'évaluation de la recherche, comme le préconise par exemple le «manifeste de Leiden » paru en avril 2015 dans la revue Nature. Signé par cinq chercheurs engagés dans les processus internationaux d'évaluation de la recherche, ce manifeste critique l'utilisation toujours plus prégnante des chiffres dans l'évaluation des travaux scientifiques, au détriment de données plus qualitatives: http:// www.leidenmanifesto.org/.

22. On en trouvera un récent exemple, parmi de très nombreux, dans le texte d'Étienne Anheim (2015), responsable éditorial de la revue des Annales, paru dans le numéro 62-4 bis de la Revue d'Histoire Moderne et Contemporaine.

23. Le cas états-unien est à cet égard éloquent, cette situation de précariat conduisant les chômeurs sortant des universités, dont celles de la Ivy League, à ne plus pouvoir rembourser les prêts conséquents qu'ils ont contractés durant leurs études supérieures. En juillet 2015, sept millions d'emprunteurs avaient ainsi cessé le remboursement de leurs créances depuis au moins une année.

24. On pourra aussi consulter l'ouvrage de Christophe Granger (2015) qui analyse notamment la multiplication et la dispersion des statuts précaires (qui confinent quelquefois au travail au noir) dans l'Université française.

25. Selon un principe bien connu que le sociologue américain Robert K. Merton (1968) a appelé le «Matthew effect » et qui tend à n'attribuer la reconnaissance scientifique qu'à certains acteurs plutôt qu'à d'autres d'après un ensemble de critères qui n'ont que peu à voir avec la différence effective de valeur scientifique. 
26. Danger que conçoivent bien, et que critiquent par ailleurs ceux-là mêmes qui, pourtant, passent à côté de cette impulsion critique que porte l'Open Access, en en restant parfois à une forme de fascination pour le simple fait que les conditions technologiques d'une plus grande circulation de l'information scientifique semblent d'elles-mêmes le justifier.

INDEX

Mots-clés : humanités numériques, économie politique, édition scientifique, libre accès, open access

\section{AUTEURS}

\section{CHRISTOPHE MAGIS}

Maître de Conférences en Sciences de l'information et de la communication, Université Paris 8 Cemti

\section{FABIEN GRANJON}

Professeur en Sciences de l'information et de la communication, Université Paris 8 - Cemti 